\title{
GLI ESOSOMI NELLA COMUNICAZIONE CELLULA-CELLULA
}

\author{
STEFANIA RAIMONDO (*) \\ Nota presentata dal m.e. Gianpiero Sironi \\ (Adunanza del 20 ottobre 2016)
}

\begin{abstract}
SUNTO. - La comunicazione cellula-cellula è un processo essenziale per il coordinamento e la corretta organizzazione dei sistemi multicellulari. Le cellule comunicano attraverso svariati meccanismi tra cui il rilascio di fattori di crescita, chemochine, piccole molecole bioattive, il contatto cellula-cellula. Negli ultimi anni è stato descritto un nuovo e sofisticato meccanismo di comunicazione cellulare basato su vescicole extracellulari. Le vescicole extracellulari sono vescicole specializzate, rilasciate nello spazio extracellulare dalla maggior parte dei tipi cellulari, in condizioni fisiologiche e patologiche. Tra i diversi sottotipi di vescicole extracellulari gli esosomi hanno recentemente ricevuto la maggior parte dell'attenzione vista la loro capacità di fungere da messaggeri nella comunicazione intercellulare.
\end{abstract}

$* * *$

ABSTRACT. - Cell to cell communication is essential for the coordination and proper organization of different cell types in multicellular systems. Cells exchange information through a multitude of mechanisms such as secreted growth factors and chemokines, small molecules (peptides, ions, bioactive lipids and nucleotides), cell-cell contact and the secretion of extracellular matrix components. Over the last few years a new and sophisticated mechanism of cell-cell communication based on extracellular vesicles has been described. Extracellular vesicles are specialized vesicles released in the extracellular space by most of cell types, under physiological and pathological conditions. Among different extracellular vesicles subtypes, exosomes $(30-100 \mathrm{~nm})$ have recently received most of the attention do to their ability to be messenger in intercellular communication.

(*) Dipartimento di Biomedicina, Neuroscienze e Diagnostica avanzata, Sezione di Biologia e Genetica, Università degli Studi di Palermo, Palermo, Italia.

E-mail: stefania.raimondo@unipa.it 


\section{LA COMUNICAZIONE CELLULA-CELLULA}

E' ormai ampiamente descritto che nessuna cellula vive isolata nel nostro organismo e che esiste una complessa rete di comunicazione tra le cellule che garantisce l'omeostasi di tessuti e organi. Le cellule comunicano e interagiscono tra loro tramite il fenomeno della segnalazione cellulare. In alcuni casi la comunicazione cellula-cellula è diretta, mediante giunzioni serrate o comunicanti, in altri casi le cellule devono poter comunicare a distanza: una cellula segnalatrice produce una molecola segnale, riconosciuta da una cellula bersaglio, per mezzo di una proteina recettore, che a sua volta produce segnali intracellulari. L'intero processo che traduce l'informazione portata dal messaggero extracellulare in cambiamenti intracellulari è chiamato trasduzione del segnale (Fig. 1). I segnali prodotti possono avere efficacia a breve o ampio raggio, in base a questo i segnali extracellulari si dividono in endocrini, paracrini e autocrini. Nella segnalazione endocrina le cellule rilasciano nel sangue sostanze dette ormoni, ovvero segnali che agiscono su cellule bersaglio lontane. Nella segnalazione paracrina, la cellula bersaglio si trova in prossimità della cellula segnale e la sostanza che funge da segnale agisce sul gruppo di cellule bersaglio adiacenti. Infine nella segnalazione autocrina le cellule agiscono contemporaneamente da segnale e da bersaglio.

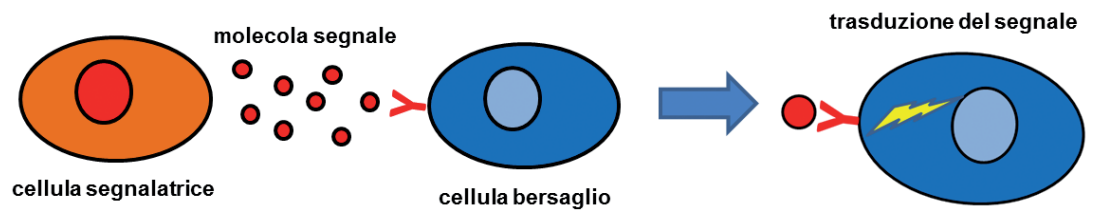

Fig. 1 - Trasduzione del segnale. Una cellula segnalatrice produce una molecola segnale che viene riconosciuta dalla cellula bersaglio per mezzo di un recettore. Il legame della molecola segnale al recettore attiva una via biochimica determinando una risposta cellulare.

\section{LE VESCICOLE EXTRA-CELLULARI}

Nell'ultimo decennio è stato descritto un nuovo meccanismo di comunicazione cellula- cellula, basato sul rilascio di un gruppo eterogeneo di vescicole lipoproteiche che fungono da veicoli di informazioni intercellulari [1] . Questi vettori, rilasciati da cellule animali e vegetali [2], e generalmente definite vescicole extracellulari, possono essere suddivisi in tre 
classi principali: esosomi, microvescicole e corpi apoptotici. Tra queste, gli esosomi hanno recentemente ricevuto maggiore attenzione. Mentre gli esosomi sono vescicole di origine endocitica, omogenee in dimensione e morfologia $(40-100 \mathrm{~nm})$, i corpi apototici sono rilasciati dalle cellule in fase di morte cellulare programmata, e infine le microvescicole derivano direttamente dalla membrana plasmatica e mostrano una dimensione variabile $(100-1000 \mathrm{~nm})$. Le vescicole extracellulari vengono inoltre denominate in base sulla base della loro origine, per esempio i prostasomi sono le vescicole secrete dalle cellule epiteliali della prostata e secrete nel liquido seminale [3], gli oncosomi quelle rilasciate dalle cellule tumorali [4].

\section{GLI ESOSOMI}

Il termine "esosomi" è stato introdotto per la prima volta nel 1983, per identificare, negli eritroblasti in via di differenziamento, le vescicole di membrane in grado di inglobare al loro interno il recettore della trasferrina (Tfr) [5]. Più recentemente la ricerca si è focalizzata su queste vescicole sia perché si sono rivelate dei veri e propri vettori di scambio di informazioni biologiche tra le cellule, sia per il loro ruolo in numerose condizioni fisiologiche e patologiche [6]. Gli esosomi sono una classe di nanoparticelle bioattive, del diametro compreso tra i 30 e i $100 \mathrm{~nm}$ e derivate dal compartimento endosomiale. Gli esosomi vengono rilasciati in vitro da diversi tipi di cellule, tra cui le cellule del sistema immunitario, le cellule staminali mesenchimali, le cellule epiteliali, le cellule endoteliali e cellule tumorali di quasi tutti gli istotipi. Inoltre gli esosomi sono stati purificati da una varietà di fluidi corporei come plasma, urina, saliva, liquido cerebrospinale, amniotico e fluido sinoviale. Gli esosomi hanno una specifica morfologia e, come indicato da analisi biochimiche e proteomiche, hanno una membrana arricchita in particolari lipidi (colesterolo, sfingomielina e ceramide) e una composizione proteica unica che li caratterizza e ne rende facile l'identificazione. Anche se gli esosomi provenienti da cellule diverse hanno una differente composizione proteica, alcune proteine sono comuni a tutti i tipi di esosomi; tra queste vi sono Alix, alcune tetraspanine e proteine chaperon. Ulteriori studi hanno inoltre evidenziato la presenza di acidi nucleici all'interno degli esosomi, quali DNA [7], mRNA [8], microRNA [9] e long non-coding RNA [10] (Fig. 2). Numerosi studi hanno dimostrato che la presenza di questi acidi nucleici all'interno degli esosomi non è casuale ma è finemente regolato da processi selettivi. 


\section{Esosoma}

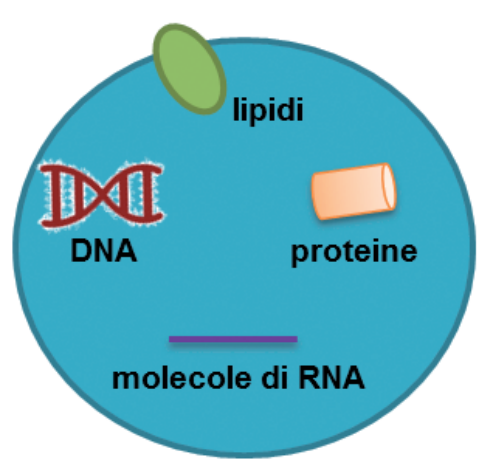

Fig. 2 - Composizione degli esosomi. Gli esosomi sono costituiti da un doppio strato lipidico, contengono proteine, metaboliti e molecole di RNA ( $m R N A$, microRNA, long noncoding RNA).

\subsection{Gli esosomi in condizioni fisiologiche e patologiche}

Gli esosomi sono ad oggi considerati parte integrante del microambiente intercellulare e possono agire come regolatori della comunicazione cellula-cellula [6]. Gli esosomi possono attivare meccanismi di trasduzione del segnale mediante interazioni fisiche ligando/recettore o mediante fusione con le cellule bersaglio e trasferimento dei loro contenuti. Possono anche essere endocitate dalle cellule bersaglio oppure rilasciare il loro contenuto nello spazio extracellulare. Essi possono agire come modulatori del sistema immunitario con effetti immunosoppressivi o promuovendone l'attivazione; sono inoltre implicati nel riciclo e smaltimento di proteine di membrana e nello scambio di materiale genetico. Una volta rilasciati dalla cellula che li produce, gli esosomi possono influenzare il comportamento della cellula bersaglio sia localmente sia a distanza attraverso i fluidi biologici. Gli esosomi sono stati purificati dal latte materno e diversi studi sottolineano come questi siano in grado di regolare l'immunità materno-fetale. Sono inoltre stati ritrovati nelle urine dove giocano un ruolo nel regolare la funzionalità renale. Un numero crescente di evidenze sperimentali sottolinea un ruolo degli esosomi nell'insorgenza e progressione di svariate patologie, quali cancro, malat- 
tie neurodegenerative, malattie infiammatorie, reumatologiche, cardiache e muscolari.

\subsection{Gli esosomi come biomarcatori}

Gli esosomi a causa della loro origine cellulare, del loro ruolo sia in condizioni fisiologiche e patologiche e dei recenti sviluppi tecnologici che consentono la loro rapida purificazione e caratterizzazione, hanno cambiato in modo significativo molte aree delle scienza clinica. I primi approcci per l'applicazione degli esosomi in diagnostica clinica risalgono a diversi anni fa. Infatti, grazie al loro contenuto proteico e di acidi nucleici, che riflette la natura e lo stato delle cellule che li rilasciano, gli esosomi sono considerati una ricca fonte di informazioni [11]. Le molecole veicolate dagli esosomi sono utili pertanto ai fini diagnostici e per il monitoraggio di trattamenti terapeutici; inoltre, il contenuto, che è ben protetto da un doppio strato lipidico che conferisce un elevato grado di stabilità, comprende alcuni biomarcatori che sarebbero altrimenti scarsamente rilevabili. Molti progressi sono stati fatti in campo oncologico, dove micro-RNA contenuti negli esosomi sono stati associati alla progressione della patologia. Ad esempio, Rabinowits e colleghi hanno dimostrato la differenza significativa in livelli di esosomi e di miRNA tra pazienti affetti da tumore al polmone e pazienti sani, suggerendo che i microRNA veicolati dagli esosomi potrebbero essere utili per lo sviluppo di test di screening per questa neoplasia [12]. Così come in ambito oncologico, gli esosomi possono rappresentare importanti biomarcatori in numerose patologie quali quelle reumatologiche [13], neurodegenerative [14] e cardiache [15]. E' ormai noto che citochine, fattori di crescita, molecole di adesione e proteine della matrice extracellulare sono secrete dalle cellule tumorali e mediano la comunicazione cellula-cellula all'interno del microambiente. Recentemente gli esosomi sono stati considerati come veicoli di queste molecole e, per questa ragione, sono considerati nuovi attori nel crosstalk tra le cellule del microambiente tumorale [16].

\subsection{Gli esosomi: un nuovo sistema di drug delivery}

Il drug delivery è lo sviluppo di sistemi alternativi di indirizzamento dei farmaci nell'organismo, con l'obiettivo si circoscriverne l'effetto biologico su una cellula bersaglio, migliorandone l'efficacia e ridu- 
cendone la tossicità. Ad oggi tante sono le informazioni riguardanti gli esosomi come sistemi di trasporto di citochine, fattori di crescita, molecole di adesione e acidi nucleici. Gli esosomi potrebbero anche essere utilizzati nel campo della terapia genica in quanto possono fungere da veicolo di composti terapeutici rappresentati da miRNA, siRNA e agenti chemioterapici [17] (Fig. 3). Di più recente interesse è l'utilizzo di esosomi come "strumenti terapeutici", in quanto recenti studi che analizzano i profili proteomici e trascrittomici di queste vescicole, hanno dimostrato che esprimono molecole sulla loro superficie, alcune delle quali si ritrovano in tutti gli esosomi (LAMP2B e CD63) mentre altre sono tessuto-specifiche e questo può essere sfruttato per indirizzare in modo specifico esosomi caricati con farmaci selettivi per determinate cellule [18]. Il drug delivery che utilizza esosomi può fornire vantaggi unici rispetto ad altri sistemi, tra cui una limitata immunogenicità ed una maggiore stabilità nel sangue. Le membrane degli esosomi possono inoltre essere modificati per mostrare epitopi che possano legarsi a specifici ligandi presenti su determinati tipi cellulari e migliorarne il delivery specifico verso tale tipo cellulare.

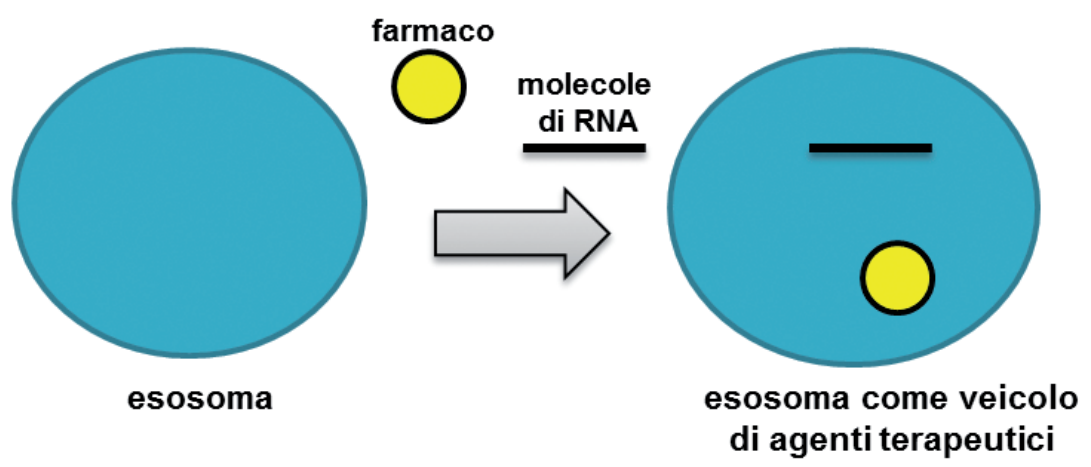

Fig. 3 - Gli esosomi come veicoli di agenti terapeutici. Gli esosomi possono essere impiegati per veicolare farmaci o molecole di RNA terapeutiche su cellule bersaglio.

\section{RINGRAZIAMENTI}

Ringrazio il Prof. Riccardo Alessandro per il supporto scientifico. Ringrazio inoltre la Fondazione Italiana per la ricerca sul Cancro (FIRC) per il finanziamento ricevuto. 


\section{BIBLIOGRAFIA}

1. Raposo G and Stoorvogel W. Extracellular vesicles: exosomes, microvesicles, and friends. J Cell Biol. 2013; 200(4):373-383.

2. Raimondo S, Naselli F, Fontana S, Monteleone F, Lo Dico A, Saieva L, Zito G, Flugy A, Manno M, Di Bella MA, De Leo G and Alessandro R. Citrus limonderived nanovesicles inhibit cancer cell proliferation and suppress CML xenograft growth by inducing TRAIL-mediated cell death. Oncotarget. 2015; 6(23):19514-19527.

3. Aalberts M, Stout TA and Stoorvogel W. Prostasomes: extracellular vesicles from the prostate. Reproduction. 2014; 147(1):R1-14.

4. Meehan B, Rak J and Di Vizio D. Oncosomes - large and small: what are they, where they came from? J Extracell Vesicles. 2016; 5:33109.

5. Harding $\mathrm{C}$, Heuser J and Stahl P. Receptor-mediated endocytosis of transferrin and recycling of the transferrin receptor in rat reticulocytes. J Cell Biol. 1983; 97(2):329-339.

6. Corrado C, Raimondo S, Chiesi A, Ciccia F, De Leo G and Alessandro R. Exosomes as intercellular signaling organelles involved in health and disease: basic science and clinical applications. Int J Mol Sci. 2013; 14(3):5338-5366.

7. Aucamp J, Bronkhorst AJ, Badenhorst CP and Pretorius PJ. A historical and evolutionary perspective on the biological significance of circulating DNA and extracellular vesicles. Cell Mol Life Sci. 2016; 73(23):4355-4381.

8. Valadi H, Ekstrom K, Bossios A, Sjostrand M, Lee JJ and Lotvall JO. Exosomemediated transfer of mRNAs and microRNAs is a novel mechanism of genetic exchange between cells. Nat Cell Biol. 2007; 9(6):654-659.

9. Thind A and Wilson C. Exosomal miRNAs as cancer biomarkers and therapeutic targets. J Extracell Vesicles. 2016; 5:31292.

10. Hewson $\mathrm{C}$ and Morris KV. Form and Function of Exosome-Associated Long Non-coding RNAs in Cancer. Curr Top Microbiol Immunol. 2016; 394: 41-56.

11. Gonzalez E and Falcon-Perez JM. Cell-derived extracellular vesicles as a platform to identify low-invasive disease biomarkers. Expert Rev Mol Diagn. 2015; 15(7):907-923.

12. Rabinowits G, Gercel-Taylor C, Day JM, Taylor DD and Kloecker GH. Exosomal microRNA: a diagnostic marker for lung cancer. Clin Lung Cancer. 2009; 10(1):42-46.

13. Withrow J, Murphy C, Liu Y, Hunter M, Fulzele S and Hamrick MW. Extracellular vesicles in the pathogenesis of rheumatoid arthritis and osteoarthritis. Arthritis Res Ther. 2016; 18(1):286.

14. Howitt J and Hill AF. Exosomes in the Pathology of Neurodegenerative Diseases. J Biol Chem. 2016; 291(52):26589-26597.

15. Kishore R, Garikipati VN and Gumpert A. Tiny Shuttles for Information Transfer: Exosomes in Cardiac Health and Disease. J Cardiovasc Transl Res. 2016; 9(3):169-175.

16. Raimondo S, Corrado C, Raimondi L, De Leo G and Alessandro R. Role of 
Extracellular Vesicles in Hematological Malignancies. Biomed Res Int. 2015; 2015:821613.

17. Kim OY, Lee J and Gho YS. Extracellular vesicle mimetics: Novel alternatives to extracellular vesicle-based theranostics, drug delivery, and vaccines. Semin Cell Dev Biol. 2016.

18. Ha D, Yang N and Nadithe V. Exosomes as therapeutic drug carriers and delivery vehicles across biological membranes: current perspectives and future challenges. Acta Pharm Sin B. 2016; 6(4):287-296. 